Relations industrielles

Industrial Relations

\title{
Code du travail - Labor Code, par Gérard Picard, Montréal 1957. 1 volume bilingue, 245 pp.
}

Volume 13, numéro 1, janvier 1958

URI : https://id.erudit.org/iderudit/1022481ar

DOI : https://doi.org/10.7202/1022481ar

Aller au sommaire du numéro

Éditeur(s)

Département des relations industrielles de l’Université Laval

ISSN

0034-379X (imprimé)

1703-8138 (numérique)

Découvrir la revue

Citer ce compte rendu

(1958). Compte rendu de [Code du travail - Labor Code, par Gérard Picard, Montréal 1957. 1 volume bilingue, 245 pp.] Relations industrielles / Industrial Relations, 13(1), 111-114. https://doi.org/10.7202/1022481ar

Tous droits réservés @ C Département des relations industrielles de l’Université Laval, 1958
Ce document est protégé par la loi sur le droit d'auteur. L'utilisation des services d'Érudit (y compris la reproduction) est assujettie à sa politique d'utilisation que vous pouvez consulter en ligne.

https://apropos.erudit.org/fr/usagers/politique-dutilisation/ 
L'étude du professeur Jamieson est d'une lecture facile. Elle a le mérite de condenser dans un nombre restreint de pages les éléments essentiels à la compréhension du mouvement ouvrier canadien. Il s'est glissé, ici et là, quelques erreurs de détail; certaines hypothèses mériteraient d'être approfondies davantage. Mais dans l'ensemble le lecteur y trouvera sûrement le meilleur essai de synthèse publié à date sur un sujet très complexe, le mouvement ouvrier canadien. L'auteur l'a bien situé dans son contexte géographique, social, économique, juridique et politique.

\section{GÉrard Dion}

\section{Mgr Guerry, La Doctrine sociale de} l'Eglise, 1 vol., 191 pp. Bonne Presse. Paris, 1957.

Les ouvrages sur la doctrine sociale de l'Eglise ne manquent pas. Plusieurs ont vu le jour en ces dernières années. Certains ont des qualités incontestées. Tels ceux du R.P. Vilain, s.j., et du R.P. Van Gester, o.p. D'autres pour diverses raisons sont moins recommandables.

Voici qu'un évêque français, bien connu en notre milieu pour ses travaux sur l'Action catholique, vient de publier en un petit volume une lettre pastorale au clergé et aux militants de son diocèse intitulée La Doctrine sociale de l'Eglise, son actualité, ses dimensions. son rayonnement.

Cet ouvrage est un des meilleurs que nous connaissons. On n'y trouve pas de «vasage », ni de jérémiades, ni de sentimentalisme. Son grand mérite est d'exprimer l'essentiel avec précision et concision. Grâce à des définitions claires et à des distinctions lumineuses, il dissipe les confusions entretenues par ceux qui, malgré leurs bonnes intentions, n'ont pas renouvelé leur vocabulaire ni assimilé les développements de l'enseignement social de l'Eglise depuis vingtcinq ans. L'auteur ne nous écrase pas non plus sous des montagnes de citations pour mieux cacher sa pensée, bien qu'il donne toujours en note les références les plus importantes.

L'ouvrage comprend d'abord une substantielle introduction où est dit en quoi consiste la doctrine sociale de
l'Eglise, pourquoi l'Eglise a une doctrine sociale, et où est fait réponse à un certain nombre d'objections. La première partie est consacrée à la conception de l'homme apportée au monde par cette doctrine. La seconde a trait à la conception de l'économie sociale. Mgr Guerry se propose de compléter son étude dans une autre étude où il exposera la conception chrétienne de la communauté humaine.

Cet ouvrage se recommande sans réserve à cause de l'autorité de son auteur et surtout à cause de ses qualités intrinsèques. Il peut fort bien être utilisé comme manuel pour un cours sur la doctrine sociale de l'Eglise. On le distribue aux Presses Universitaires Laval, Québec.

GÉrard Dion

Code du travail - Labor Code, par Gérard Picard, Montréal 1957. 1 volume bilingue, 245 pp.

Le président général de la CTCC vient de publier sous ce titre un essai de codification de la législation du travail pour la province de Québec. Il y exprime sa propre conception de la législation du travail ainsi que ce qu'elle doit, selon lui, comprendre. Il le fait en tenant compte des mesures législatives existantes et surtout des pratiques courantes en notre pays et de sa longue expérience dans lorganisation syndicale et la négociation de conventions collectives. Voilà pourquoi dans son essai on trouvera plusieurs suggestions que l'auteur croit devoir offrir. Comme il serait trop long de faire une étude critique de chacune des innovations, il vaut mieux se contenter de les présenter aux lecteurs, quitte à revenir plus tard sur l'une ou l'autre des originalités de ce projet. Les voici.

Le Code du Travail érige la législation du travail en un système juridique particulier et remplace les lois éparses et fragmentaires édictées depuis environ un demi-siècle.

Le Code du Travail prévoit qu'il doit y avoir consultation officielle des associations patronales et des organisations syndicales de travailleurs avant la formation de tout organisme consultatif, administratif ou semi-judiciaire relevant de la juridiction du Ministre du Travail. 
Voici quelques cas concrets d'application. Actuellement tous les membres de la Commission de relations ouvrières, de la Commission du salaire minimum et de la Commission des Accidents du Travail sont nommés par le gouvernement et deviennent, à compter de leur nomination, des fonctionnaires du service civil. Avec l'application du Code du Travail, les présidents des commissions seraient nommés par le gouvernement, mais les membres ne seraient nommés qu'après consultation officielle des associations patronales et des organisations syndicales de travailleurs. De plus les membres seraient, en nombre égal, des représentants patronaux et des représentants syndicaux et ils ne deviendraient pas des fonctionnaires du service civil. Ainsi, il y aurait consultation officielle, représentation officielle et participation à l'élaboration ou à l'application, selon le cas, de la législation du travail. Les divers organismes seraient donc des organismes tripartites avec représentation du gouvernement, des associations patronales et des organisations syndicales de travailleurs comme la méthode en a été suggérée depuis 1919 par le Traité de Versailles.

Le Code du Travail vise à protéger efficacement l'exercice du droit d'association des travailleurs et interdit, par des mesures efficaces, les «unions de compagnies », les 《syndicats de boutiques ». Le Code protège les travailleurs contre les congédiements et les suspensions pour activités syndicales. Des mesures sévères et rapides d'application seraient appliquées contre les employeurs qui se rendraient coupables de représailles contre des travailleurs syndiqués en plus d'être obligés de les réinstaller dans leurs fonctions.

Le Code du Travail, entre autres innovations, accorde aux fonctionnaires provinciaux, aux instituteurs et institutrices, aux contremaîtres, aux professionnels salariés, les mêmes droits d'association et de négociations collectives qu'aux autres travailleurs.

Le Code reconnaît et légalise toutes les mesures de sécurité syndicale et fournit des notes explicatives sur les diverses formes connues de sécurité syndicale.

Le Code du Travail établit un nouveau régime de négociations collectives.
Dans ce nouveau régíme, le Code fixe d'abord la définition de la convention collective. Voici les deux articles quj contiennent cette définition:

«La convention collective de travail est une entente écrite relative aux salaires, aux conditions de travail et d'emploi, intervenue entre, d'une part, un employeur, et d'autre part, une organisation syndicale de travailleurs. La convention collective peut également être conclue entre, d'une part, plusieurs employeurs, une ou plusieurs organisations d'employeurs, et d'autre part, une ou plusieurs organisation syndicales de travailleurs.

«La convention peut régir les systèmes de rendement, les pensions de retraite, les plans d'assurances, les prestations supplémentaires aux prestations d'assurance-chômage, et régir toute autre mesure de sécurité sociale et de bien-être sur lesquelles les parties tombent d'accord. »

Que se passera-t-il lorsque, dans une entreprise, un syndicat a obtenu l'adhésion de la majorité absolue des travailleurs? Le syndicat n'a qu'à se présenter au bureau de l'employeur, lui donner la preuve qu'il est majoritaire, et l'employeur est tenu de le reconnaître. Ii n'est plus question, comme règle générale, du certificat de reconnaissance syndicale. Le syndicat peut également exiger, dès ce moment, la retenue des cotisations syndicales en produisant les autorisations individuelles de ses membres, et l'employeur doit faire cette retenue sur la paye gratuitement. Les parties peuvent s'entendre à l'amiable sur les fonctions à inclure et à exclure de l'unité de négociations et cette entente est valide. Les parties n'iront devant la Commission des relations de travail que s'il y a doute sur la majorité ou si elles ne peuvent s'entendre sur les fonctions à inclure ou à exclure de l'unité de négociations.

Un syndicat directement reconnu par l'employeur ou accrédité par la Commission des relations de travail sont sur un pied d'égalité.

Il découle de ce qui précède qu'un syndicat majoritaire peut exiger la retenue des cotisations syndicales avant de commencer les négociations et il 
peut, lors des négociations, préciser quelle est la formule de sécurité syndicale qu'il a l'intention de discuter. Aucune mesure de sécurité syndicale n'est interdite.

Le Code fixe une période de deux mois pour les négociations directes, après quoi le syndicat peut recourir à la grève. Il peut aussi, s'il le désire, recourir à la conciliation ou à l'arbitrage, mais ces deux procédures deviennent des procédures volontaires.

Dans les quinze jours de la date où il a été reconnu directement ou accrédité par la Commission des relations de travail, le syndicat doit soumettre par écrit à l'employeur son projet ảe convention collective ou ses demandes. Dans les quinze jours suivants l'employeur doit soumettre ses contre-propositions par écrit. Les parties se rencontrent à leur convenance, mais si après deux mois de négociations, à compter de la date où le syndicat a soumis ses demandes, il n'y a pas entente, les travailleurs peuvent recourir à la grève.

L'employeur doit fournir au syndicat majoritaire les noms, occupations et salaires de tous les travailleurs de i'unité de négociations.

Le Code du Travail n'interdit la grève que dans les quelques rares cas où des vies humaines pourraient être en danger c'est-à-dire que la grève est interdite aux policiers, aux pompiers, aux infirmiers et infirmières des hôpitaux, aux gardiens des prisons communes et des asiles d'aliénés. Mais des recours efficaces sont prévus pour ces catégories de travailleurs pour compenser la perte du droit de grève.

Le Code du Travail ne traite pas de la grève pour indiquer que cette matière relève des provinces. Le droit de grève relève du droit criminel canadien. Mais le Code traite de ce sujet strictement en vue de poser les limites acceptables à l'exercice de ce droit.

Le Code établit également que la rétroactivité des augmentations des salaires sera fixée de la date où un syndicat a formulé ses demandes, s'il s'agit de la négociation d'une première convention collective, et de la date d'expiration de la convention précédente, s'il de travailleurs.

s'agit de remplacer cette convention par une autre.

La convention peut être conclue pour toute période ne dépassant pas trois ans. Mais quelle que soit la période pour laquelle la convention a été conclue les parties peuvent convenir qu'elle sera continuée à la condition qu'une partie puisse $y$ mettre fin en aucun temps en donnant à l'autre partie un avis écrit de trente jours.

Au cas de grève, il est interdit à l'employeur de recourir à des briseurs de grève.

A la fin d'une grève, tous les grévistes doivent être repris au travail à leurs occupations respectives.

La Commission des relations de travail n'a aucune autorité pour décider de la légalité ou de l'illégalité d'une grève et elle n'a aucune juridiction pour enlever à un syndicat, par suite d'une grève, son droit de représentation et de régociations.

Le Code du Travail maintient les décrets et comités paritaires, mais apporte des précisions de nature à ne plus confondre les conventions collectives et les décrets.

Le Code du Travail prévoit aue le gouvernement de la Province de Québec et ses ministères, de même que les commissions qui en relèvent, ne peuvent accorder de contrats de construction, de fabrication ou de fournitures qu'à des employeurs signataires de conventions collectives de travail avec des syndicats reconnus ou accrédités.

Parmi les innovations du Code du Travail, il faut noter l'établissement des tribunaux du travail et la nomination d'une Magistrature du Travail. Ces tribunaux du travail auront juridiction sur toutes les questions de travail qui, actuelement, sont réglées par la Cour Supérieure, la Cour des Sessions de la Paix ou la Cour du Magistrat. La procédure, devant les tribunaux du travail, sera simple, rapide et peu dispendieuse. Les tribunaux du travail seront présidés par des juges, mais ces derniers auront à leurs côtés, pour les aviser, des assesseurs désignés par les associations patronales et les organisations syndicales 
Le Code innove également en matière de formation professionnelle, d'orientation professionnelle et d'apprentissage.

Enfin, le Code du Travail établit une Régie des prix dont la juridiction s'étend aux producteurs primaires (à l'exception des cultivateurs), aux fabricants et aux grossistes. Il ne s'agit pas d'un organisme de contrôle des prix, ni d'une système d'arbitrage obligatoire des prix, mais d'un organisme permanent d'enquête dont le but est d'éclairer l'opinion publique en matière de prix.

Le Code du Travail est en vente au bureau de M. Gérard Picand, 8227, boulevard St-Laurent, Montréal.

Administrative Action, by William $\mathbf{H}$. Newman. N.Y., Prentice-Hall, 1950, pp. 460.

Tel que suggéré par l'entête, ce volume traite des principes et techniques d'une administration dynamique. Il s'adresse spécialement aux administrateurs des grandes corporations modernes sans toutefois exclure ceux des entreprises plus modestes, en leur fournissant les moyens conceptuels et pratiques de s'adapter aux changements techniques et sociaux afin de préserver et promouvoir les valeurs propres à notre civilisation occidentale contemporaine. "Administrative Action 》 s'avèrera très utile au personnel de direction, car il contient une analyse pénétrante et compréhensive des différentes fonctions administratives, et apporte de nouvelles idées pour améliorer les procédures de direction.

Le livre se divise en cinq parties, dont chacune discute l'un des éléments de base de toute administration: la planification, l'organisation, l'assemblement des ressources, la direction et le contrôle.

L'administration d'une entreprise exige avant tout de planifier les opérations, budgets, standards, etc. Le responsable sera plus efficace s'il connaît bien les diverses formes de planification, et s'il peut choisir la meilleure se- lon les caractéristiques des phénomènes et les conditions ambiantes. Dans les chapitres deux à cinq, la nature, les avantages et les désavantages des types de planification utilisés sont décrits. Les deux chapitres suivants exposent comment prendre les décisions dont dépendent les succès ou la faillite des programmes, même les mieux conçus.

La deuxième partie sur l'organisation est assurément la meilleure de l'ouvrage. L'auteur lui consacre d'ailleurs la plus large part de son espace. D'un côté, les éléments de toute organisation y sont individuellement analysés de façon claire et détaillée. Newman couvre à tour de rôle la division départementale, la division des services, la délégation d'autorité, le rôle des spécialistes (staff), les comités, la décentralisation, les bureaux de direction et la représentation des intérêts. A notre avis, les chapitres sur la délégation de l'autorité et le «staff» méritent une mention particulière, et beaucoup d'administrateurs auraient profit à les lire et suivre leurs enseignements. Cette partie se termine par une synthèse de l'organisation structurelle qui permet de considérer les éléments de l'organisation comme un tout intégré.

Les deux parties qui suivent sont plus faibles et moins élaborées que les précédentes. L'une traite de la détermination des besoins de personnel, du choix, de l'évaluation, de la formatiors et de la rémunération des administrateurs. L'autre analyse brièvement la direction et la coordination à travers la supervision. La dernière partie porte sur les étapes du contrôle, avec un chapitre spécial sur le contrôle budgétaire, l'un des instruments les plus efficaces de la direction, et se termine par une analyse de la motivation qui exprime le besoin d'une meilleure compréhension de la motivation humaine dans le processus administratif.

«Administrative Action》 est un bon livre qui vaut mieux que les manuels habituellement servis. Il ne déparera pas les rayons de celui qui s'intéresse aux questions administratives.

L.-M. Tremblay 